\title{
Right-Arm Robotic-Aided-Therapy with the Light-Exoskeleton: A General Overview
}

\author{
Luis I. Lugo-Villeda, Antonio Frisoli, Edoardo Sotgiu, \\ Giovanni Greco, and Massimo Bergamasco \\ Perceptual Robotics, Scuola Superiore Sant'Anna, via Rinaldo Piaggio, 34, \\ 56025 - Pontedera (Pisa), Italy \\ \{1.lugovilleda, a.frisoli, massimo.bergamasco\}asssup.it
}

\begin{abstract}
Rehabilitation robotics applications and their developments have been spreading out as consequences of the actual needs in the human activities of daily living (ADL). Exoskeletons for rehabilitation are one of them, whose intrinsic characteristics are quite useful for applications where repetitive, robustness and accurate performance are a must. As a part of robotic-mediatedrehabilitation programme into the worldwide, the exoskeletons are trying to improve the ADL of disable people through the fusion of several disciplines that lets to expand the capabilities of wearing a powered robotic exoskeletal device for rehabilitation tasks. This fact deserves to present this contribution from a general scope point of view, i.e., the technologies integration and its associated knowledge. So far, the Light-Exoskeleton which is intended for human arm rehabilitation in post-stroke patients is introduced. Preliminary experimental results as well as the involved stages about the system show the capabilities of using a robotic-constrained-rehabilitation for human arm.
\end{abstract}

Keywords: Exoskeletons, Robotic-Aided-Rehabilitation, Emerging Technologies Integration, Robotic Constrained Therapy.

\section{Introduction}

Robotic-Aided-Rehabilitation is one of the most multidisciplinary emerging fields that has been studied for several decades; it is due to the synergetic ability to use the interaction of robots into the disable humans-workspace as part of the rehabilitation robotics realm [4]. Actually, rehabilitation robotics belongs to the so-called program Health Workforce of the WHO (World Health Organization) wherein this technology is one of the forces driving such a program [12]. According to U.S. Centers for Disease Control and Prevention and the American Heart Association [2], 80,000,000 American adults (one in three) have one or more types of cardiovascular disease (CVD), of whom $38,100,000$ are estimated to be age 60 or older, this represents as total direct and indirect amount of CVD for 2008 of $\$ 475.3$ billion. Similar conditions are prevailing in Italy and EU countries; therefore, boosting the application of rehabilitation robotics for assisting people to recover their normal daily-living skills is quite essential. But what does rehabilitation robotics involve? The meaningful answer in our context leads to define it as follows:

L.M. Camarinha-Matos, P. Pereira, and L. Ribeiro (Eds.): DoCEIS 2010, IFIP AICT 314, pp. 205-214, 2010. (C) IFIP International Federation for Information Processing 2010 
Rehabilitation Robotics is the multi-disciplinary field which concerns with restoring a disable person to an acceptable level of whether physical, mental or even social interaction with him/her environment by means of applied robotics, emerging technologies and health care areas.

This definition helps to assess the robotic-mediated-therapy as an area of rehabilitation robotics, offering the capabilities to improve the gradual outcome of post-stroke patients engaged on rehabilitation of neuromotor control in comparison with conventional therapy [14]. Also the idea of robotic-mediated-therapy is an opportunity to innovate and improve trough new emerging fields the rehabilitation endeavour field. Here, we present some advantages by using our upper limb rehabilitation demonstrator in comparison with other approaches.

\section{Contribution to Technological Innovation}

Despite of these impressive achievements regarding exoskeletons, it has been said, they are in the early stage due to the involved fields that need being enhanced [15]. Nevertheless, as long as we unify important concepts with their associated technology, new trends along the technological innovation stream are arising for exploiting at maximum the application of such devices into rehabilitation fields.

Particularly, two of the foremost goals are presented as innovative:

1. The Robotic-Constrained-Therapy (RCT) which has been conceptualised as the application of constrained kinematics and constrained dynamics into a framework for treating the problem of upper limbs plasticity at the level of arm's joints.

2. The inclusion of nonlinear controllers that our best knowledge, they are practically unknown, but those present important characteristics managing the total energy of the closed-loop to be passive in every time and controlling the parametric and non parametric uncertainties induced by the patient's tremor.

\subsection{Contribution to Technological Innovation Trough Emerging Fields}

The contribution of this article is three-fold:

1. A robotic-aided-rehabilitation platform is generally presented under the rehabilitation robotics philosophy.

2. A general landscape of the novelty robotic constrained therapy is introduced.

3. Preliminary results are promising, such that the proposed platform is ready to undergo to real disable patients at the Cisanello hospital, Pisa, Italy, as the immediate future work.

\subsection{Organization}

The outline of this contribution is organized as follows. Section 3 shows the actual work into this field. The proposed system under the robotics rehabilitation trends is given into Section 4. Besides this a set of experimental results on healthy people is shown into Section 5. Section 6 presents, a set of discussions about the obtained results. Finally the conclusions and future work are given in Section 7. 


\section{Related Work}

We describe the actual work driven by two major key approaches [11]:

\subsection{Fixed-Frame Exoskeletons}

In the first classification, we have the Armin II, an exoskeleton for human-arm rehabilitation whose 6 DoF have been used in conjunction with virtual environments, suitable for helping post-stroke patients. The exoskeleton uses a simple PD plus gravity compensation controller to obtain apparently interactive forces, which are computed on the basis of the virtual environment interaction by an impedance controller [5]. A 7-DoF upper-limb exoskeleton which can be used for therapeutic diagnostics and for physiotherapy or as a haptic device in virtual reality simulation is presented in [6], however it does not introduces neither the techniques for doing rehabilitation nor the control scheme for assuring stability ${ }^{1}$.

\subsection{End-Effector Devices}

In the second category, we have more devices to analyse, but all of them are very competitive. Mostly of end-effector devices have been gone under the commercial track, that is the case of the MIT Manus[7], A pantograph-based manipulator which works into 2-D, it is constituted by a Stanford manipulator which trains patients who have suffered an stroke and have lost arm motor skills. The main purpose of MITManus is guiding the human-arm and works in conjunction with a virtual environment for displaying the desired and real reaching exercise carried out by the patient. Another example is the Mirror Image Movement Enabler system, [8], a PUMA 560based robot, which can impose bilateral 3-D force-position motions. Likewise, the Bi-manu-track robot for upper-limbs rehabilitation uses active practise of forearm movements, such as pronation/supination, and wrist flexion/extension into mirror like fashion [9]. EU Gentle/s Project presents a 3 degree-of-freedom (DoF) haptic device for right-handed subjects, which uses virtual environments for reaching exercises to tackle the arm tracking problem by using a path planning based on smooth polynomials [10]. Finally, ARM-guide [11] drives the forearm along a linear position profile into 2-D space having guided force training in joint space. It is clear that in robotic-mediated-rehabilitation devices either exoskeletons or end-effector devices, the virtual reality technology enhances the performance of upper-limb poststroke rehabilitation [3].

\section{The Rehabilitation Robotics Platform}

This section is devoted to present our proposed platform for carrying out the arm therapy by using a right-arm exoskeleton.

\footnotetext{
${ }^{1}$ Notice that both exoskeletons belong to fixed-inertial frame exoskeletons.
} 


\subsection{The Light-Exoskeleton Architecture}

The general scheme is presented in the Fig. 1.b). The system is clearly composed by the Light-Exoskeleton and its hardware/software integration; the therapist that handles the therapy session uses a workstation which contains the set of projected scenarios into the screen, following the clinical protocol and giving instructions to the patient during the rehabilitation sessions. The Light-Exoskeleton ${ }^{2}$ is a right-arm assistant device, it is composed of five degree-of-freedom (DoF), it is capable of feeding the patient-arm with bio-mechanical force-feedback through the four-active DoF (using four-PWM controlled motors), and the last DoF is used for computing the exerted force direction in the operational space.

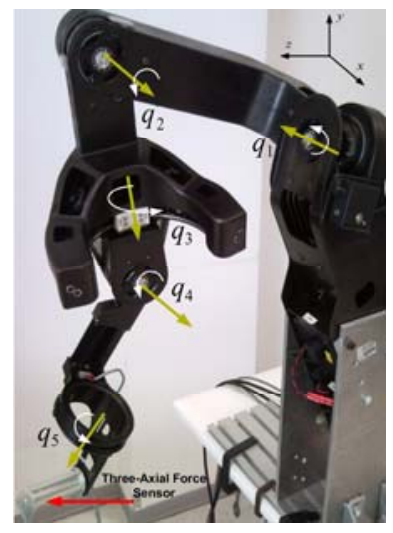

a)

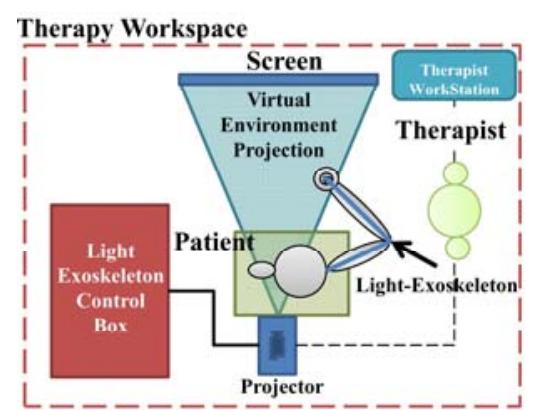

b)

Fig. 1. The right-arm robotics rehabilitation platform. a) Light-exoskeleton architecture. b) Upper-part rehabilitation workspace.

The corresponding generalized coordinates are measured by digital encoders such that the vector $\mathbf{q}=\left\{q_{1}, q_{2}, q_{3}, q_{4}, q_{5} \in \mathbb{R}\right\}$ corresponds with the human arm motions, i.e., adduction/abduction, flexion/extension, internal/external rotation, forearm flexion/extension and finally pronation/supination. In addition, one important variable is defined in order to analyse the arm's behaviour, the triplet $\mathbf{X}_{h} \in \mathbb{R}^{3}$ which stands as the temporal space variable of hand-palm; that vector is mapped onto the virtual scenario workspace for the interaction with video-game-like exercises. The exerted force by the patient's hand-palm is measured by means of a three-axial $100 \mathrm{~N}$ force sensor, $\lambda=\left\{\lambda_{x}, \lambda_{y}, \lambda_{z} \in \mathbb{R}\right\}$, see Fig. 1.a). One of the foremost goals of this platform is helping post—stroke (acute mild to moderate) patients handling their arm-stiffness and recovering the neurocontrol of their arm using the constrained kinematics and dynamics; also the brain plasticity through several kinesthetic coordination exercises is treated.

\footnotetext{
${ }^{2}$ Hereafter Light-Exoskeleton could be called for short L-Exos.
} 


\subsection{Involved Technologies, Knowledge and Their Trends to the Innovation}

The performance of the rehabilitation platform depends on the application of several important areas such that innovative concepts and paradigms arise depending on the defined outcomes into arm rehabilitation as well as the patient's conditions. The set of relevant areas are described as follows.

- Applied Robotics. This field is quite important because trough the constrained kinematics and constrained dynamics we can handle the patient's limbs to move either a maximum or minimum angular displacement depending on the clinical protocol; for instance, the weighted-iterative inverse kinematics lets to get working more the internal/external rotation than arm adduction/abduction, but maintaining the angular position set $\mathbf{q} \in \mathbb{R}^{5}$ in the Jacobian-null-space, whereas the dynamics of the system is modeled as a Differential-Algebraic-Equation (DAE index-2) yielded by the Lagrange formalism [13], representing the motion equations of the exoskeleton in nonfree motion, i.e., including the contact of L-Exos with the patient. Likewise, these approaches are attempting in the best case to emulate the occupational therapist in contact with the patient arm; regulating force and position simultaneously or separately depending on the clinical procedure.

- Applied Nonlinear Control. This field is required and innovative into this platform; because of the applications of several nonlinear controllers have demonstrated better performance than linear ones as used on the related work [15]. The whole energy of the system should remain passive (controlled) all time, and these nonlinear controllers gather that condition. Also, the free-model nonlinear controllers are desirable and largely unknown at our best knowledge; it is due to many exoskeletons for rehabilitation have been designed for neuroscience or biomechanical groups, and with only a proportional plus derivative with gravity compensation controllers are used but neglecting all parametric non-modeled patient-induced uncertainties. Therefore, the next set of operation modes have it bases on the nonlinear controllers design:

1. Position Mode. This mode lets the robot to take the control of the upper limbs transferring their position and perhaps their orientation; the patientlimbs are passive.

2. Triggered-Gains Position Mode. In this mode, the patient starts applying a force for triggering the motion; the exerted force by the patient modules the percentage of the robot's help, adjusting the gains of the position controller which tracks the imposed trajectory in the task.

3. Composed Force/position Control Mode. The patient is constrained by a defined task in terms of kinesthetic forces and positions onto his/her workspace; it induces active resistance to patient improving in this manner the patient coordination effort.

4. Direct Force Mode. The patients can freely move their arm into a defined space wherein just the exerted force is controlled.

5. Open Loop Mode. This option lets to patient interacting with the virtual environment trough several video-game-like applications; the patients use their actual skills for playing games but exerting forces and positions of their limbs according the game responses. 


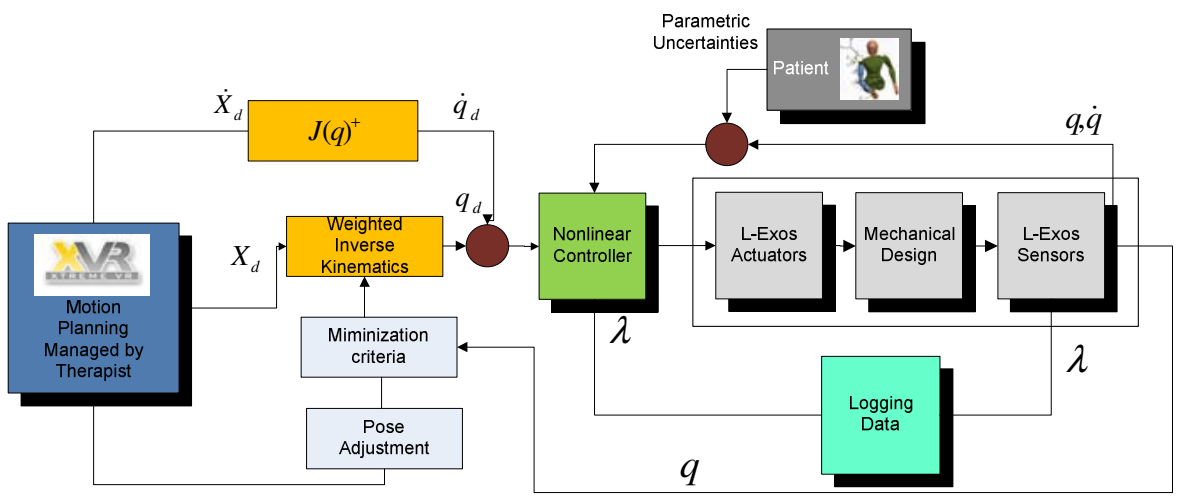

Fig. 2. The general closed-loop scheme, the path planning is generated according the virtual reality application into XVR software; afterwards the kinematics and dynamics establish the rehabilitation operation modes trough nonlinear controller described above.

- Virtual Environments. The virtual scenarios render more interactively the rehabilitation, attracting the full concentration of the patient, at the degree that they feel very comfortable in front of video-game-like applications for carrying out the session. Those scenarios include shapes to be tracked into spatial coordinates within the video-game-like virtual reality application, wherein the therapist can vary parameters like, the target distance, the number of trials, the timing, the maximum force, the depth, see Figure 3.

- Bio-Signals: The analysis of other signals coming from the muscles(EMG) different from the system's states are a good option for measuring the effectiveness and progress of the therapy[16]; even we could know whether the exoskeleton is not wellaligned with the patient-arm rotation axes.

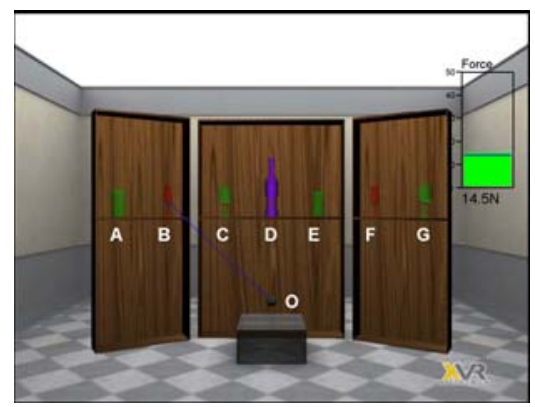

a)

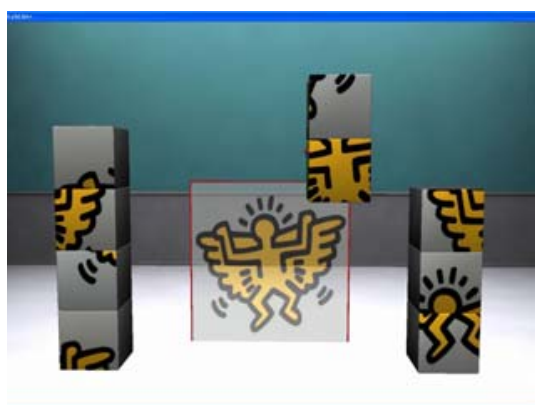

b)

Fig. 3. Virtual reality applications with force sensing. a) The reaching video-game-like application, it will be used for our trials. b) Cubes application, it consists into a 3-dimensional puzzle to be organised by the patients. 
- Medical Assessment: It includes several scales for assessing a rehabilitation session:

a) The Fugl-Meyer scale, b) Modified Ashworth scale, (c) Range of Motion, etc.

- Robotic Constrained Therapy (RCT): The main idea of this statement is to constrain from the kinematical and dynamical point of view the right-arm joints, such as the real therapist does; The magnitude of range motion depends on the clinical protocol and it is directly programmed and adjusted into the GUI (graphical user interface) of the therapist's workstation as long as the patient is able to perform an exercise.

\section{Preliminary Experimental Results}

Some preliminary experiments were carried out by a healthy people; nevertheless, similar results are expected on real disable people. We have chosen the presented task of the Fig.3.a) which is one of the most used tasks into rehabilitation, the reaching task. It consists into tracking from initial point to the final point and vice versa a spatial target in a determined lapse; in this experiment we used the first kind of operation mode ${ }^{3}$ a virtual scenario and the minimum-jerk-criterion for the path planning. Firstly, notice that in Fig.4 the constrained kinematics is working with the same desired target but with different weights of shoulder joints; the main outcome relies on getting adduction/abduction arm motion to be minimum when varying its weight, $\mathrm{w}_{1}=1, \mathrm{w}_{1}=50, \mathrm{w}_{1}=100$; but the arm requires more effort for reaching the target on the external/internal rotation; thus constraining the first joint motion. Secondly, Fig. 5 shows the reaching task into spatial coordinates in terms of the handpalm position $\mathbf{X}_{h} \in \mathbb{R}^{3}$ for one target, the constraining planes work like big potential fields that return the hand-palm of the patient when it tries to go away from the

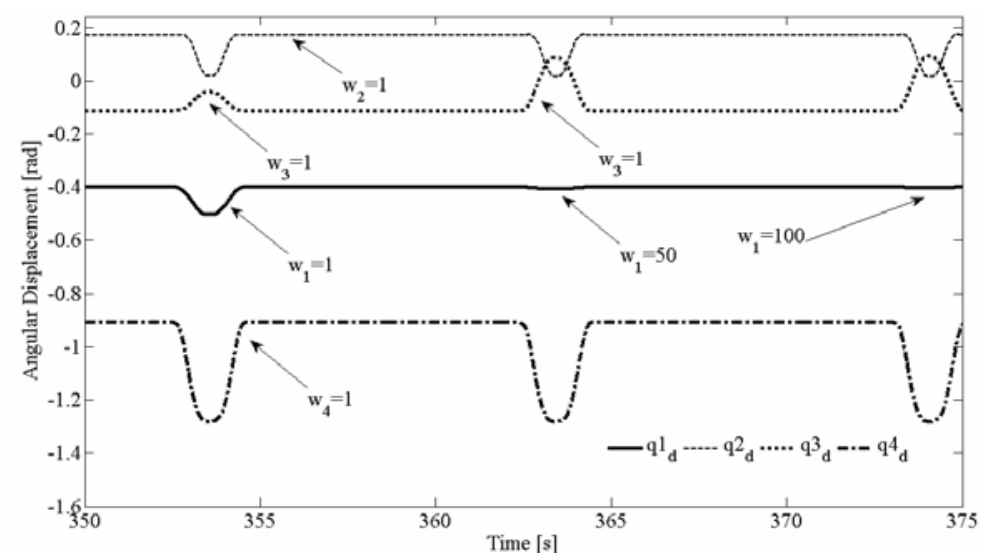

Fig. 4. The constrained kinematics using the weighted iterative inverse kinematics. Notice that the adduction/abduction is working less as long as its weight is bigger.

\footnotetext{
${ }^{3}$ The remaining operation modes are working with healthy people but not presented here.
} 


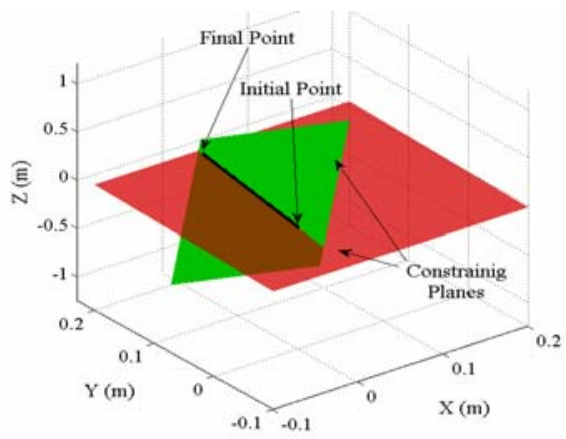

Fig. 5. The constrained dynamics is used into the nonlinear controller; we have constrained the hand-palm to move only onto the desired trajectory by introducing two constraining planes
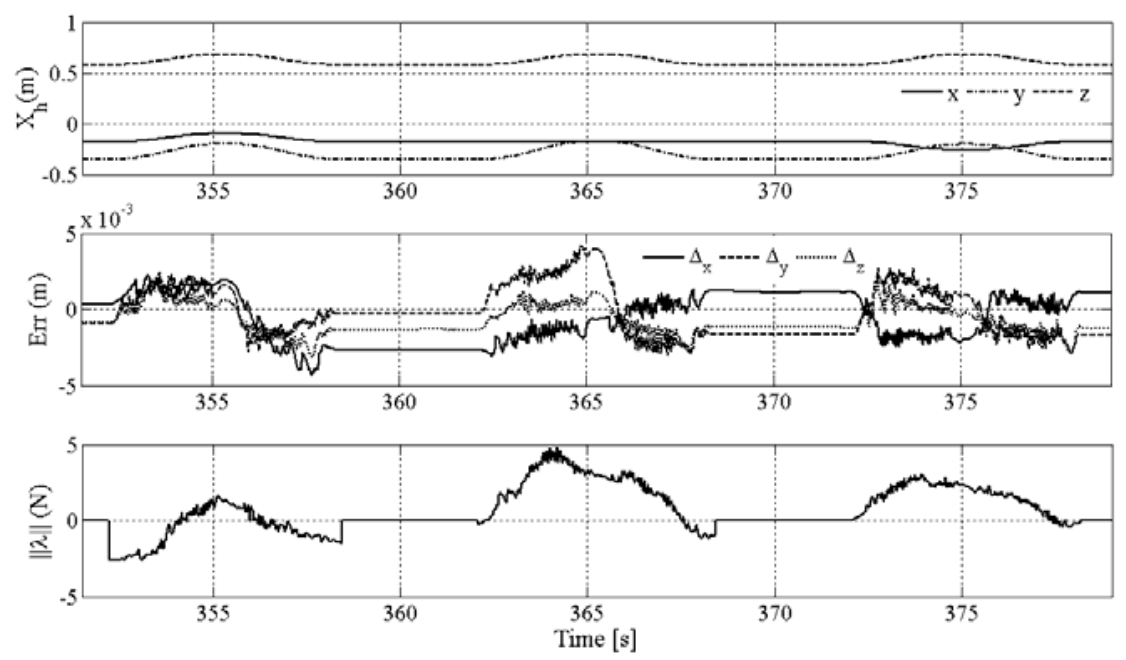

Fig. 6. Experimental results of the hand-palm of the patient, the Cartesian error and the exerted force along the trajectory during three-reaching targets in arm rehabilitation

desired position. Finally for three targets the desired and actual position, the low spatial errors, and the exerted force $\lambda \in \mathbb{R}^{3}$ along the desired trajectories have shown in Fig.6. Notice the exerted force during reaching task is not more than 5 Newton; we used the same position mode for all experiments.

\section{Discussions}

Until now, this contribution remains on the edge of performing the proposed approach on healthy patients and the other control modes deserve more attention; nowadays clinical pilot trials are actively working. The importance of presenting the required 
tools is a must. It is clear that many drawbacks are still being a challenge, because of the complexity of the L-Exos and the different behaviour of the patient to patient. The performance of getting a successful rehabilitation platform obeys to the correct use of the involved technologies and its associated knowledge; to our best knowledge, the constrained robotics presents the advantages so clear with respect to other works.

\section{Conclusions and Future Work}

We are presenting the involved disciplines for having stable right-arm rehabilitation. We introduce the Light-Exoskeleton which is intended for human arm rehabilitation suitable for post-stroke patients who present problems with normal arm skill motions. Several kinds of operation modes in non free motion make the system robust, accessible and feasible to be used in disable people. The constrained kinematics and dynamics foster the arm therapy to the robotic constrained therapy as innovative concept; thereby the robot represents the physician as long as we draw advantages from the emerging fields. Preliminary experimental results are shown as well as the involved stages about the system. The neuroplasticity can be managed via kinesthetic channel and coordinated motions imposed by the L-Exos, but always under the supervision of the physician or therapist. As near future work, the application on real patients is actually working as an eight-month clinical programme for assisting arm impaired people at Cisanello Hospital, Pisa, Italy. Also different scenarios are being programmed according to patients needs.

\section{References}

1. Hillman, M.: Rehabilitation robotics from past to present a historical perspective. In: Bien, Z.Z., Stefanov, D. (eds.) Advances in Rehabilitation Robotics. LNCIS, vol. 306, pp. 2544. Springer, Heidelberg (2004)

2. Organization, W.H.: The world health report 2006: working together for health, Technical report 924156317 6, WHO (2006)

3. The American Heart Association: Heart disease \& stroke statistics, our guide to current statistics and the supplement to our heart \& stroke facts 2009 update. Technical Report 1, American Heart Association (2009)

4. Prange, G.B., Jannink, M.J., Groothuis-Oudshoorn, C.G., Hermens, H.J., Ijzerman, M.J.: Systematic review of the effect of robot-aided therapy on recovery of the hemiparetic arm after stroke. J. Rehabil. Res. Dev. 43(2), 171-184 (2006)

5. Nef, T., Mihelj, M., Kiefer, G., Perndl, C., Muller, R., Riener, R.: Armin - exoskeleton for arm therapy in stroke patients. In: IEEE 10th International Conference on Rehabilitation Robotics, ICORR 2007, pp. 68-74 (2007)

6. Perry, J., Rosen, J.: Design of a 7 degree-of-freedom upper-limb powered exoskeleton. In: The First IEEE/RAS EMBS International Conference on Biomedical Robotics and Biomechatronics, BioRob 2006, pp. 805-810 (2006)

7. Krebs, H.I., Hogan, N., Volpe, B.T., Aisen, M.L., Diels, C.: Overview of clinical trials with mit-manus: a robot-aided neuro-rehabilitation facility. Technol. Health Care 7(6), 419-423 (1999) 
8. Lum, P., Burgar, C., Van der Loos, M., Shor, P., Majmundar, M., Yap, R.: The mime robotic system for upper-limb neuro-rehabilitation: results from a clinical trial in subacute stroke. In: 9th International Conference on Rehabilitation Robotics, ICORR 2005, June-1 July 2005, pp. 511-514 (2005)

9. Schmidt, H., Hesse, S., Werner, C., Bardeleben, A.: Upper and lower extremity robotic devices to promote motor recovery after stroke -recent developments. In: 26th Annual International Conference of the IEEE Engineering in Medicine and Biology Society, IEMBS 2004, vol. 2, pp. 4825-4828 (2004)

10. Amirabdollahian, F., Loureiro, R., Harwin, W.: A case study on the effects of a haptic interface on human arm movements with implications for rehabilitation robotics. In: Proceedings of 1st Cambridge Workshop on Universal Access and Assistive Technology (CWUAAT) incorporating 4th Cambridge Workshop on Rehabilitation Robotics), pp. 17-24 (2002)

11. Reinkensmeyer, D., Dewald, J., Rymer, W.: Guidance-based quantification of arm impairment following brain injury: a pilot study. IEEE Transactions on Rehabilitation Engineering 7(1), 1-11 (1999)

12. Burdea, G.: Keynote address: Virtual rehabilitation-benefits and challenges. In: 1st International Workshop on Virtual Reality Rehabilitation (Mental Health, Neurological, Physical, Vocational) VRMHR 2002 Lausanne, Switzerland, pp. 1-11 (2002)

13. Spong, M.W., Hutchinson, S., Vidyasagar, M.: Robot Modeling and Control, 1st edn. John Wiley and Sons, USA (2006)

14. Lugo-Villeda, L.I., Frisoli, A., Parra-Vega, V., Bergamasco, M.: Robust tracking of the light-exoskeleton for arm rehabilitation tasks. In: IEEE-IFAC 9th International Symposium of Robot Control 2009, pp. 823-828 (2009)

15. Lugo-Villeda, L.I., Frisoli, A., Parra-Vega, V., Bergamasco, M.: Regressor-free force/position control of fixed-base exoskeletons for rehabilitation tasks. In: Conference on Intelligent RObots and Systems. The 2009 IEEE/RSJ International (IROS 2009), pp. 1639-1645 (2009)

16. Lugo-Villeda, L.I., Frisoli, A.,, Sandoval-Gonzalez, B.M., et al.: Haptic Guidance of Light-Exoskeleton for Arm-Rehabilitation Tasks. In: Symposium on Human Interactive Communication (RO-MAN 2009). The 8th IEEE/RAS International, p. 189 (2009) 